\title{
HIGHLIGHTS
}

HYPERTENSION

\section{Elevated systolic blood pressure in middle age is associated with late-life dementia}

Epidemiological studies have shown that, in addition to being a risk factor for cardiovascular and cerebrovascular diseases, hypertension is associated with the onset of dementia. Lenore Launer and colleagues have now reported that a quarter of cases of late-life dementia in a cohort of Japanese-American men could be prevented by keeping systolic blood pressure (BP) values below $120 \mathrm{mmHg}$ in middle age. "This is excellent news because it suggests one way to prevent a [devastating] disease," says Dr Launer.

Age-related cognitive decline is distressing for both the patient and their family, and has considerable economic and societal consequences. As there are no proven therapies for established dementia, targeting of risk factors is vitally important. Although our understanding of the mechanisms linking hypertension and dementia is still evolving, it is clear that dementia is a progressive illness that can begin many years before clinical symptoms manifest.

Launer et al. investigated data from 7,878 Japanese-Americans who were enrolled in the Honolulu Heart Program (HHP) and its follow-up investigationthe Honolulu Asia Aging Study.

Participants were born between 1900 and 1919 and were, therefore, in middle age (mean 54 years) at HHP baseline in 1965. As the investigators write, "the long follow-up, serial standardized assessment of BP [and] dementia ... provides a unique opportunity to estimate the impact on dementia rates of reducing BP levels."

At HHP enrollment, $41.3 \%$ and $37.4 \%$ of men had systolic prehypertension (120-140 $\mathrm{mmHg}$ ) and hypertension (>140 mmHg), respectively. During follow-up, 491 cases of dementia (6.2\%) were recorded. After adjustment for confounding factors, the population attributable risk for dementia with midlife systolic BP values $\geq 120 \mathrm{mmHg}$ was

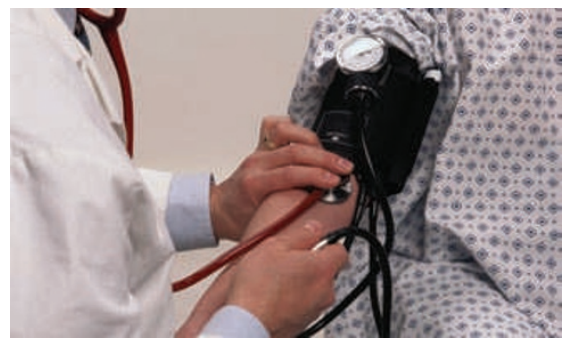

$27 \%$ among those who did not receive antihypertensive medication. "The next step ... is to study in a clinical trial whether the effectiveness of lowering BP to $120 \mathrm{mmHg}$ reduces the risk of cognitive decline and dementia," concludes Launer.

Alexandra King

Original article Launer, L. J. et al. Lowering midlife levels of systolic blood pressure as a public health strategy to reduce late-life dementia: perspective from the Honolulu Heart Program/Honolulu Asia Aging Study. Hypertension 55, 1352-1359 (2010) 